I NVESTI GACIÓN

\title{
Evaluación económica de la incapacidad laboral temporal en una institución de salud
}

\section{Economic assessment of temporary work disability in a health care institution}

\author{
Antonio Castillo Guzmán'; Carmen Arocha Mariño"; Nurys B. Armas \\ Rojas'"'; Digna Camejo Esquijarosa'v
}

\author{
' Máster en Salud Pública. Profesor Auxiliar. Instituto de Cardiología y Cirugía \\ Cardiovascular. La Habana, Cuba. \\ "Doctora en Ciencias de la Salud. Profesor Titular. Escuela Nacional de Salud \\ Pública. La Habana, Cuba. \\ III Especialista de I Grado en Epidemiología. Instructor. Instituto de Cardiología y \\ Cirugía Cardiovascular. La Habana, Cuba. \\ IV Máster en Educación Médica. Asistente. Escuela Nacional de Salud Pública. La \\ Habana, Cuba.
}

\section{RESUMEN}

Introducción La seguridad social cubana garantiza una cobertura total a toda la población desde el triunfo de la Revolución. La incapacidad laboral temporal es un aspecto que el sistema actual de seguridad social contempla. El Anexo II de la Resolución Ministerial 176/89 regula la prescripción, por los médicos, de los días de reposo.

Objetivos Valorar la repercusión económica, para el Instituto de Cardiología y Cirugía Cardiovascular, del pago por los certificados médicos presentados por sus trabajadores en el año 2005.

Métodos Se realizó una evaluación económica parcial del tipo descripción de costos. Fueron revisados los 224 certificados médicos recibidos en el centro en ese año. Se confrontó la incapacidad laboral temporal prescrita con lo establecido y se cuantificaron las diferencias.

Resultados Los certificados médicos recibidos prescribieron 523 días más que lo reglamentado, en términos económicos significó el pago de 4 286,03 pesos por encima de lo estipulado.

Conclusiones Se indican más días de reposo por certificados médicos que lo autorizado por la Resolución Ministerial 176/89. Se considera que el Anexo II tiene 17 años de emitido y debe ser revisado y ampliado el espectro diagnóstico.

Palabras clave: Seguridad social, incapacidad laboral temporal, evaluación económica, certificado médico.

\section{ABSTRACT}


Introduction Since the triumph of the revolution, Cuban social welfare assures overall coverage to the whole population. Temporary work disability is an issue that the present social welfare system takes into account. Annex 2 to the ministerial resolution no.176 of 1989 stipulates the prescription by physicians of the number of days that an individual under treatment should rest.

Objectives To assess the economic impact of the payment of medical leaves submitted by sick workers at the Institute of Cardiovascular Surgery and Cardiology in 2005.

Methods Partial cost description-type economic assessment was made in which all the 224 medical leaves submitted that year were checked. The prescribed temporary work disability was compared with that of the resolution, and then differences were estimated.

Results Medical leaves prescribed 523 days more than the number set by the law, which economically accounted for the payment of 4286,03 pesos in excess.

Conclusions The number of rest days prescribed in medical leaves was higher than the number stipulated by the ministerial resolution no.176 of 1989. It was considered that Annex 2 was issued 17 years ago; therefore, the diagnostic range should be reviewed and expanded.

Key words: Social welfare, temporary work disability, economic assessment, medical leave.

\section{NTRODUCCI ÓN}

La atención a la salud del pueblo es un principio que los líderes del estado cubano tuvieron presente desde antes del triunfo de la Revolución. En el juicio por el asalto al cuartel Moncada de Santiago de Cuba el 26 de julio de 1953, el Dr. Fidel Castro manifestó: "El problema de la tierra, el problema de la industrialización, el problema de la vivienda, el problema del desempleo, el problema de la educación y el problema de la salud del pueblo; he ahí concretados los seis puntos a cuya solución se hubieran encaminado resueltamente nuestros esfuerzos..." 1

El joven gobierno revolucionario instalado en los primeros días de enero de 1959 encontró que la mayor parte de las cajas de retiro y jubilaciones habían sido defalcadas. En ese mismo año, hizo que estas fueran dotadas de fondos para cumplir con los compromisos establecidos, y se elevó, además, el mínimo de jubilaciones y pensiones a 40 pesos mensuales. ${ }^{2}$ A finales del año 1958 existían 52 cajas de retiro y seguros sociales que cubrían riesgos de invalidez, muerte, vejez, accidentes de trabajo y enfermedades profesionales. ${ }^{3}$ La realidad es que, la efectividad del seguro de enfermedad y de accidente era muy limitada, quedando parte de la fuerza de trabajo fuera del sistema, además, los desempleados no tenían protección alguna.

Por ley, los patronos tenían que contratar la cobertura con las compañías de seguro en los casos de enfermedades profesionales y accidentes del trabajo, estando obligadas, aquellas, a facilitar la atención médica, hospitalaria y de farmacia, sustituyendo el salario por una dieta hasta por un año, llegando a ser una renta vitalicia en el caso de incapacidad total. Otra cosa ocurría en la realidad, los 
afectados, para alcanzar esos beneficios, se veían obligados a emprender una demanda judicial, cuyo costo corría a cuenta de sus bolsillos, lo que, en caso de un fallo a su favor disminuía el ingreso que debía percibir por concepto de indemnización.

En relación con el seguro de maternidad, existían amplias disposiciones extendidas a toda la población trabajadora, pero estas solo cubrían a las personas radicadas en las capitales de provincia, pues los servicios se concentraban en las ciudades más importantes.

Los seguros por invalidez, vejez y muerte se fueron aplicando con diferentes criterios, sin uniformidad, variando incluso, la cotización entre el 3 y el $10 \%$ de los salarios. ${ }^{3}$ Más de la mitad de los trabajadores se quedaron fuera del sistema y, por lo general, al cambiar de actividad se perdían los derechos al retiro acumulado de la anterior ocupación. ${ }^{4}$

El seguro por enfermedad solo cubría a los empleados de la administración pública, que recibían el salario completo el primer mes de licencia por enfermedad y la mitad el segundo. Podían estar ausentes del trabajo por esta causa hasta seis meses pero sin recibir subsidio alguno. Los obreros, por causa de enfermedad, tenían derecho a recibir, por lo general, tres días de trabajo al mes, con un límite máximo de nueve días al año. ${ }^{5}$

El sistema actual de seguridad social se ha ido perfeccionando en forma progresiva y continua. Ya en los primeros años del gobierno revolucionario se adoptaron medidas para lograr uniformidad y racionalización, como fue la creación del Banco de Seguro Social que asumió las obligaciones para indemnizaciones por accidentes de trabajo que con anterioridad estaban a cargo de las compañías de seguro privadas.

En la década de los años 60, la medida de carácter general más importante fue la Ley No. 1100 de Seguridad Social de 27 de marzo de 1963 a partir de la cual se consolida la nueva estructura del sistema en tres principios: a) la unidad administrativa de la seguridad social, b) la participación de los trabajadores en el procedimiento para conceder las prestaciones y c) considerar el gasto total de la seguridad social como una partida del presupuesto nacional. ${ }^{6}$

Con posterioridad, otros instrumentos legales se han ido incorporando al actual sistema, entre ellos se destacan: la Ley de Maternidad, que ha sufrido ventajosas modificaciones a lo largo del tiempo para la madre trabajadora; el régimen de prestaciones ordinarias de largo plazo, que beneficia a hombres y mujeres trabajadores con 60 años o más los primeros, y 55 o más años las segundas, que deseen retirarse; jubilaciones por incapacidad; la viudez; el régimen de prestaciones ordinarias de corto plazo que establece los subsidios por enfermedad y accidentes común; los subsidios por enfermedad y accidentes profesionales y otros.

Una medida del crecimiento y extensión de la seguridad social cubana es el hecho siguiente: en 1959 el número de beneficiarios era de 145 000, en el año 2003 fue de 1 859, 870 ciudadanos, los egresos en prestaciones monetarias, en ese mismo período se elevaron de \$114 millones a \$2 561 millones. El Estado Cubano destina actualmente a la seguridad social más del $11 \%$ de su producto interno bruto. ${ }^{3,7}$

Como parte de la seguridad social, la incapacidad laboral temporal, en particular, ha sido objeto de atención por el Estado Revolucionario. La Resolución No. 170 de 16 de septiembre de 1988 del Ministerio de Salud Pública (MINSAP) fue un paso 
adelante para mejorar las normas que regían la expedición y control de los certificados médicos (CM). En su segundo por cuanto señala "....la necesidad de establecer mecanismos de control efectivos sobre la expedición de los certificados médicos, con el objetivo de evitar su utilización incorrecta en detrimento del régimen de seguridad social." 8

Posteriormente la Resolución Ministerial No. 176 de 1989 (que derogó la anterior) y su Anexo II, significó un avance para perfeccionar la expedición y control de los CM y para solucionar dificultades detectadas. El artículo 11 de esta establece que: "El facultativo en el momento de prescribir los días de invalidez del trabajador viene obligado a otorgar como límite máximo de días en cada certificado, los que aparecen en el listado de categoría de enfermedades, como anexo II de la presente Resolución." 9

Ante esta normativa, los autores del presente trabajo se interesaron en conocer su forma de aplicación, y en una reciente visita a las provincias de Pinar del Río, La Habana y Ciudad de La Habana, formando parte de un equipo de supervisión del MINSAP, y a título personal, uno de ellos entrevistó a un grupo de médicos [ 12 del nivel primario, 16 del secundario y 26 especialistas y 20 residentes del Instituto de Cardiología y Cirugía Cardiovascular (ICCCV)] para explorar si conocían el Anexo II de dicha resolución. El resultado fue que solo dos, que son dirigentes de un policlínico la conocían, y ninguno la consultaba para prescribir la incapacidad laboral temporal. También, como parte de la investigación realizada, se constató que algunos trabajadores del ICCCV con reposo prescrito, en ocasiones acuden a la dirección del centro solicitando que se les certifique que están recuperados del problema de salud que tenían, antes de cumplirse el tiempo de la invalidez temporal declarado, lo que hizo pensar en la existencia de contradicciones entre lo establecido por el MINSAP y lo prescrito por algunos médicos.

Teniendo en cuenta estas consideraciones, resultó interesante conocer, ¿en qué medida los CM que amparan la incapacidad laboral temporal de los trabajadores del ICCCV fueron emitidos teniendo en cuenta lo dispuesto en el Anexo II de la Resolución del MINSAP que está vigente? ¿Existen diferencias importantes entre las prescripciones y lo establecido? ¿A cuánto ascienden los pagos por este concepto? Las repuestas a estas interrogantes constituyen los objetivos de esta investigación que servirán tanto a la dirección del ICCCV como al MI NSAP para considerar la realización de una revisión del grado de vigencia del anexo a la resolución citada y de su aplicación, y poder corregir sus posibles insuficiencias para evitar de este modo los posibles excesos en los pagos, o por el contrario, que se prescriba menor invalidez temporal que la que necesitan los enfermos, con perjuicio a su salud.

\section{MÉTODOS}

Se realizó una evaluación económica parcial del tipo descripción de costos según Drummond y otros ${ }^{10}$ de la incapacidad laboral temporal en el ICCCV en el período comprendido entre el primero de enero y el treinta y uno de diciembre del año 2005.

Se revisaron $224 \mathrm{CM}$ que constituyeron la totalidad de los certificados recibidos en el departamento de recursos humanos durante el tiempo elegido para la investigación. Las variables seleccionadas fueron: edad, sexo, clasificación laboral, diagnóstico, días de reposo prescritos por el médico, centro emisor y dinero pagado. 
Los diagnósticos fueron clasificados en 10 grupos escogidos según la Guía para la certificación de días de reposo, Anexo II de la Resolución Ministerial 176/89 sobre los Certificados Médicos, del MINSAP ${ }^{11}$ agregándole los CM amparados por el Decreto Ley No. 234 de la Maternidad de la trabajadora, de fecha 13 de agosto del 2003 y por la Resolución No. 22 del 13 de octubre del 2003. El monto de la seguridad social por cada CM se calculó según el artículo 25 de La ley número 24 de Seguridad Social que comenzó a regir el primero de enero de $1980 .{ }^{12}$

Se calculó la diferencia entre días de reposo prescritos realmente y la propuesta por la guía del MINSAP, se cuantificó en pesos cubanos esa diferencia. Como medida de resumen se empleó el porcentaje.

\section{RESULTADOS}

Del total de CM recibidos en el departamento de recursos humanos del ICCCV, el $75,9 \%$ correspondió al sexo femenino y el $24,1 \%$ al sexo masculino. Los grupos de edades con más afectaciones fueron: de 25 a 34 años con el 29,5\%, de 35 a 44 años con el 24,1 \% y las edades de 45 a 54 años con el 22,3 \% (tabla 1 ).

Tabla 1. Certificados médicos por sexo y grupos de edades. ICCCV 2005

\begin{tabular}{|c|c|c|c|c|}
\hline \multirow{2}{*}{ Edad } & \multicolumn{2}{|c|}{ Sexo } & \multirow{2}{*}{ Total } & \multirow{2}{*}{$\%$} \\
\hline & $\mathrm{M}$ & $\mathrm{F}$ & & \\
\hline $15-24$ & 4 & 31 & 35 & 15,6 \\
\hline $25-34$ & 12 & 54 & 66 & 29,5 \\
\hline $35-44$ & 14 & 40 & 54 & 24,1 \\
\hline $45-54$ & 18 & 32 & 50 & 22,3 \\
\hline $55-64$ & 4 & 11 & 15 & 6,7 \\
\hline+65 & 2 & 2 & 4 & 1,8 \\
\hline Total & 54 & 170 & 224 & 100,0 \\
\hline
\end{tabular}

Teniendo en cuenta la clasificación laboral escogida, el personal de enfermería aportó el 46,4\% del total de los CM, siguiéndoles los técnicos con el 23,2\% (tabla 2). 
Tabla 2. Certificados médicos según clasificación laboral de los pacientes. ICCCV 2005

\begin{tabular}{|c|c|c|c|c|}
\hline \multirow{2}{*}{ Clasificación laboral } & \multicolumn{2}{|c|}{ Sexo } & \multirow[b]{2}{*}{ Total } & \multirow[b]{2}{*}{$\%$} \\
\hline & $M$ & $\mathrm{~F}$ & & \\
\hline Enfermeras & 22 & 82 & 104 & 46,4 \\
\hline Médicos & 5 & 3 & 8 & 3,6 \\
\hline Técnicos & 11 & 41 & 52 & 23,2 \\
\hline Administrativos & 4 & 15 & 19 & 8,5 \\
\hline Servicio & 12 & 29 & 41 & 18,3 \\
\hline Total & 54 & 170 & 224 & 100,0 \\
\hline
\end{tabular}

El promedio de trabajadores enfermos por mes fue de 18,6 . Abril, con 27 , fue el mes con más afectaciones (figura).

Los hospitales fueron los centros que emitieron el mayor número de CM con el 48,7 $\%$, seguido de los policlínicos con el 39,7\%.

Las enfermedades del aparato osteomioarticular y del tejido conjuntivo, incluyendo los traumatismos, con los diagnósticos de sacrolumbalgia y artrosis, en ese orden, fueron los más frecuentes en este grupo, representaron el $26,3 \%$ de la morbilidad total y ocasionaron el mayor número de días de incapacidad laboral temporal con el $31,8 \%$ seguidos de las enfermedades del aparato genitourinario con el 10,1\%.

La comparación de los días prescritos con lo establecido en la mencionada guía, arrojó una diferencia de 523 días de más a favor de los primeros (tabla 3).

Tabla 3. Certificados médicos, días de invalidez temporal prescritos y máximo de días según Anexo II Resolución 176/89. ICCCV 2005

\begin{tabular}{|c|c|c|c|c|c|c|}
\hline \multirow[t]{2}{*}{$\begin{array}{c}\text { Categorías de las } \\
\text { enfermedades }\end{array}$} & \multicolumn{2}{|c|}{$\begin{array}{l}\text { Certificados } \\
\text { médicos }\end{array}$} & \multicolumn{2}{|c|}{$\begin{array}{l}\text { Días de } \\
\text { invalidez } \\
\text { según } \\
\text { certificado } \\
\text { médico }(A)\end{array}$} & \multirow{2}{*}{\begin{tabular}{|l|} 
Máximo \\
de días de \\
invalidez \\
según \\
Anexo II \\
Resolución \\
$176 / 89$ \\
(B)
\end{tabular}} & \multirow{2}{*}{$\begin{array}{c}\text { Diferencia } \\
(A-B)\end{array}$} \\
\hline & No. & $\%$ & No. & $\%$ & & \\
\hline $\begin{array}{l}\text { Enfermedades } \\
\text { del aparato } \\
\text { osteomioarticular } \\
\text { y del tejido } \\
\text { conjuntivo } \\
\text { (incluye } \\
\text { traumatismo) }\end{array}$ & 59 & 26,3 & 960 & 31,9 & 915 & $45(+)$ \\
\hline $\begin{array}{l}\text { Enfermedades } \\
\text { del aparato }\end{array}$ & 46 & 20,5 & 620 & 20,5 & 316 & $304(+)$ \\
\hline
\end{tabular}




\begin{tabular}{|c|c|c|c|c|c|c|}
\hline genitourinario & & & & & & \\
\hline $\begin{array}{l}\text { Enfermedades } \\
\text { del aparato } \\
\text { digestivo }\end{array}$ & 14 & 6,3 & 312 & 10,3 & 407 & $95(-)$ \\
\hline $\begin{array}{l}\text { Enfermedades } \\
\text { del sistema } \\
\text { nervioso y } \\
\text { órganos de los } \\
\text { sentidos }\end{array}$ & 10 & 4,5 & 237 & 7,8 & 216 & $21(+)$ \\
\hline $\begin{array}{l}\text { Intervenciones } \\
\text { quirúrgicas } \\
\text { (todos los tipos) }\end{array}$ & 8 & 3,6 & 220 & 7,3 & 125 & $95(+)$ \\
\hline $\begin{array}{l}\text { Enfermedades } \\
\text { del aparato } \\
\text { respiratorio }\end{array}$ & 15 & 6,7 & 181 & 6,0 & 124 & $57(+)$ \\
\hline $\begin{array}{l}\text { Trastornos } \\
\text { mentales }\end{array}$ & 7 & 3,1 & 165 & 5,5 & 98 & $67(+)$ \\
\hline $\begin{array}{l}\text { Enfermedades } \\
\text { de la piel y del } \\
\text { tejido celular } \\
\text { subcutáneo }\end{array}$ & 11 & 4,9 & 139 & 4,6 & 94 & $45(+)$ \\
\hline $\begin{array}{l}\text { Enfermedades } \\
\text { del aparato } \\
\text { circulatorio }\end{array}$ & 7 & 3,1 & 108 & 3,6 & 109 & $1(-)$ \\
\hline $\begin{array}{l}\text { Enfermedades } \\
\text { de las glándulas } \\
\text { endocrinas, de la } \\
\text { nutrición, del } \\
\text { metabolismo y } \\
\text { trastornos de la } \\
\text { inmunidad }\end{array}$ & 3 & 1,3 & 75 & 2,5 & 90 & $15(-)$ \\
\hline Subtotal & 180 & 80,3 & 3017 & 100,0 & 2494 & $523(+)$ \\
\hline $\begin{array}{l}\text { Embarazo, parto, } \\
\text { puerperio }\end{array}$ & 19 & 8,5 & 756 & - & 75 & - \\
\hline $\begin{array}{l}\text { Resolución } \\
\text { embarazo }\end{array}$ & 25 & 11,2 & 780 & - & 780 & - \\
\hline Subtotal & 44 & 19,7 & & & & \\
\hline Total & 224 & 100,0 & 4553 & 100,0 & 4030 & $523(+)$ \\
\hline
\end{tabular}

El ICCV pagó \$21 61009 por concepto de incapacidad laboral temporal en el año analizado contra $\$ 17342,06$ que debía haber pagado, es decir, el 19,8 \% más que si se hubiera aplicado lo establecido en la regulación existente (tabla 4).

Tabla 4. I mporte pagado por certificados médicos y propuesta según lista de categorías. ICCCV 2005

\begin{tabular}{|c|c|c|c|}
\hline $\begin{array}{c}\text { Categorías de las } \\
\text { enfermedades }\end{array}$ & $\begin{array}{c}\text { Importe }(\$) \\
\text { de los } \\
\text { certificados }\end{array}$ & $\begin{array}{c}\text { Importe }(\$) \\
\text { de los } \\
\text { certificados }\end{array}$ & $\begin{array}{c}\text { Diferencia } \\
(\mathrm{A}-\mathrm{B})\end{array}$ \\
\hline
\end{tabular}




\begin{tabular}{|c|c|c|c|}
\hline & $\begin{array}{l}\text { médicos Real } \\
\text { (A) }\end{array}$ & $\begin{array}{l}\text { médicos. } \\
\text { Anexo II } \\
\text { Resolución } \\
\text { (B) }\end{array}$ & \\
\hline $\begin{array}{l}\text { Enfermedades del } \\
\text { aparato osteomioarticular } \\
\text { y del tejido conjuntivo } \\
\text { (incluye traumatismo) }\end{array}$ & 7289,15 & 6048,28 & $\begin{array}{c}1240,87 \\
(+)\end{array}$ \\
\hline $\begin{array}{l}\text { Enfermedades del } \\
\text { aparato genitourinario }\end{array}$ & 4758,75 & 2292,08 & $\begin{array}{c}2466,67 \\
(+)\end{array}$ \\
\hline $\begin{array}{l}\text { Enfermedades del } \\
\text { aparato digestivo }\end{array}$ & 2163,98 & 2649,84 & $\begin{array}{c}485,86(- \\
)\end{array}$ \\
\hline $\begin{array}{l}\text { Enfermedades del } \\
\text { sistema nervioso y } \\
\text { órganos de los sentidos }\end{array}$ & 1441,78 & 1233,06 & $\begin{array}{c}208,72 \\
(+)\end{array}$ \\
\hline $\begin{array}{l}\text { Intervenciones } \\
\text { quirúrgicas (todos los } \\
\text { tipos) }\end{array}$ & 1605,54 & 1517,70 & $87,84(+)$ \\
\hline $\begin{array}{l}\text { Enfermedades del } \\
\text { aparato respiratorio }\end{array}$ & 863,89 & 591,75 & $\begin{array}{c}272,14 \\
(+)\end{array}$ \\
\hline Trastornos mentales & 1144,13 & 654,78 & $\begin{array}{c}489,35 \\
(+)\end{array}$ \\
\hline $\begin{array}{l}\text { Enfermedades de la piel } \\
\text { y del tejido celular } \\
\text { subcutáneo }\end{array}$ & 902,89 & 573,94 & $\begin{array}{c}328,95 \\
(+)\end{array}$ \\
\hline $\begin{array}{l}\text { Enfermedades del } \\
\text { aparato circulatorio }\end{array}$ & 903,33 & 1045,93 & $\begin{array}{c}142,60(- \\
)\end{array}$ \\
\hline $\begin{array}{l}\text { Enfermedades de las } \\
\text { glándulas endocrinas, de } \\
\text { la nutrición, del } \\
\text { metabolismo y trastornos } \\
\text { de la inmunidad }\end{array}$ & 536,65 & 734,70 & $\begin{array}{c}198,05 \\
)\end{array}$ \\
\hline Subtotal & 21610,09 & 17342,06 & \\
\hline $\begin{array}{l}\text { Embarazo, parto, } \\
\text { puerperio }\end{array}$ & 8062,50 & 8062,50 & - \\
\hline Resolución embarazo & 5922,24 & 5922,24 & - \\
\hline Subtotal & 13984,74 & 13984,74 & - \\
\hline Total & 35594,83 & 31326,80 & 4268,03 \\
\hline
\end{tabular}

Los pagos relacionados con el embarazo, parto y puerperio así como los pagos a madres con niños menores de 1 año que se acogen a las leyes vigentes fue de \$13 984,74 lo que representó el $39,3 \%$ de los pagos por seguridad social.

\section{DISCUSIÓN}


Los servicios médicos, la incapacidad laboral, la invalidez, las pérdidas en la producción y los días de servicios dejados de prestar por una reducción de la capacidad de trabajo temporal y/o la mortalidad prematura, como consecuencia de una enfermedad, tienen una repercusión económica sobre el presupuesto del estado. ${ }^{13} \mathrm{El}$ modo en que se cubren estos gastos guarda relación con el modelo económico y las políticas sociales de los gobiernos. Las posibilidades que brinda el sistema de seguridad social en Cuba, con un modelo económico socialista y con políticas sociales, no deben ser mal utilizadas ya que las pérdidas económicas que eso ocasiona impiden la inversión de esos recursos en el desarrollo del país en general.

El $58 \%$ de los trabajadores del ICCCV son del sexo femenino, si se tiene en cuenta que de los $224 \mathrm{CM}$ recibidos, 45 fueron por embarazo, parto, puerperio o licencias posnatales, la contribución de este sexo se reduce entonces del 75,9 al 55,8 \% ya que esas causas no son enfermedades sino estados fisiológicos.

Por otro lado, la administración siempre ha estado pendiente de las semanas de receso escolar (febrero, abril y noviembre), y de los meses de julio y agosto, período de vacaciones escolares, en que los trabajadores que son madres y no tienen otros familiares para que les cuide a sus hijos durante ese tiempo, supuestamente presentan CM como un medio para garantizar la atención a aquellos y no incurrir en indisciplinas laborales. La distribución temporal de los CM rompió ese mito, pues, salvo en el mes de abril en que hubo un pico (aunque coincidió con una de las etapas de receso escolar), en el resto del tiempo se comportó aproximadamente igual incluyendo los meses de vacaciones escolares.

El hecho de que el mayor porcentaje de CM fuera emitido por los hospitales y no por los policlínicos puede estar relacionado con el nivel de resolutividad entre estos dos niveles a favor del primero.

La concentración del 53,6 \% de la morbilidad en las edades comprendidas entre los 25 y los 44 años resulta paradójica, toda vez que son las edades menos sensibles a las enfermedades. En cuanto a las enfermedades osteomioarticulares y del tejido conjuntivo (incluyendo los traumatismos) que contribuyeron con el $26,3 \%$ del total, con relación al reposo, debe ser el mínimo que permita el dolor y durar como máximo 2 días $^{14}$ y no el encontrado en este estudio que osciló entre 7 y 30 días. A una sola persona se le indicaron 96 días de reposo por esta causa, de forma consecutiva. La guía citada establece hasta 14 días de reposo para la sacrolumbalgia, luego entonces, siempre que se prescribió por encima de esta cifra se cometió una violación, lo más probable, por desconocimiento de las regulaciones existentes.

La artrosis es más frecuente en las personas mayores de 45 años, observándose un incremento de hasta el $30 \%$ entre las edades de 45 a 64 años y hasta el $68 \%$ por encima de los 65 años de edad. ${ }^{15}$ Varios autores señalan que el factor de riesgo más importante para las artropatías es la edad. ${ }^{15-17}$ Álvarez Cambra afirma que: "Esta enfermedad se presenta en la edad media y senectud". ${ }^{18}$ Debe precisarse con más atención su diagnóstico en las edades inferiores a las informadas internacionalmente, aunque autores como Kenneth D. Brandt plantee que existe una prevalencia de hasta el $10 \%$ en sujetos de entre 15 y 24 años de edad. ${ }^{19}$

En el caso de los días de incapacidad por enfermedades del aparato digestivo, circulatorio y de las enfermedades de las glándulas endocrinas, de la nutrición, del metabolismo y trastornos de la inmunidad, las prescripciones fueron menores que lo normado, esto puede ser perjudicial para la salud de los enfermos al no recibir 
los cuidados considerados imprescindibles para su restablecimiento. Claro está que no se pretende, con este análisis convertir a la guía en una horma rígida.

El pago del 19,8 \% por encima de lo debido constituye un uso inadecuado del presupuesto destinado al pago de la seguridad social.

El monto pagado por la seguridad social no es discutible siempre que el mismo se ajuste a lo que establece la ley, esto no significa que se justifique una erogación superior sin una razón convincente, que nunca será la no aplicación de la legislación vigente. Por otro lado, el Anexo II de la Resolución citada tiene 17 años de emitido, ignora la introducción de medios terapéuticos modernos como los centros de rehabilitación, entre otros, no recoge un gran número de diagnósticos que son causa de morbilidad, es dudoso el conocimiento y su uso por los encargados de aplicarla, por lo que queda, a la apreciación del médico, la prescripción de invalidez temporal sin un criterio colegiado entre expertos.

\section{Agradecimientos}

A Bárbara Muñoz Donis secretaria de la Vicedirección de Asistencia Médica por su contribución en la transcripción de este trabajo. A las compañeras Bárbara Mena Miranda, trabajadora social, que intervino en la elaboración del listado de los certificados médicos recibidos en el año 2005 y María Regla González Landa del Departamento de Recursos Humanos, que fue muy amable al permitirme revisar las Leyes, Decretos-Leyes y Resoluciones relacionados con el tema de este artículo.

\section{REFERENCI AS BI BLI OGRÁFICAS}

1. Castro F. La Historia me absolverá. La Habana. Editorial Ciencias Sociales; 1957.

2. Castro F. Primer Congreso del partido Comunista de Cuba. Informe Central. 2da ed. La Habana: Departamento de Orientación Revolucionaria del Comité Central del Partido Comunista de Cuba; 1975.

3. Cuba: estilo de desarrollo y políticas sociales. CEPAL. México: Siglo XXI; 1980.

4. Rodríguez J L, Carriazo G. Erradicación de la pobreza en Cuba. La Habana: Editorial Ciencias Sociales; 1987.

5. Cuba. Decreto 798 de 13 abril de 1938. Trabajo. Contratos referentes al trabajo. Reglamento. Gaceta Oficial de 27 de mayo de 1938 (copia corregida).

6. Cuba. Ley 1100 de 27 marzo de 1963. Gaceta Oficial de 14 de abril de 1963.

7. Morales A. Batalla de ideas, empleo y seguridad social (II y final). Granma. Año 40 número 57. 2 da ed., 6 de marzo del 2004, p.3.

8. MINSAP. Resolución No. 170-88. Reglamento para la Expedición y Control de los Certificados Médicos. 16 de Septiembre de 1988. La Habana: MINSAP; 1988.

9. Resolución No. 176-89. Reglamento para la Expedición y Control de los Certificados Médicos. 4 de septiembre de 1989. La Habana: MINSAP; 1989. 
10. Drummond MF, O'Brien BJ, Stoddart GL, Torrance GW. Métodos para la Evaluación Económica de los Programas de Asistencia Sanitaria. 2da ed. Madrid: Díaz de Santos; 2001.

11. MINSAP. Sobre certificados médicos. Guía para certificación de días de reposo. Ministerio de Salud Pública. Anexo II. Resolución Ministerial 176/89. La Habana: MINSAP; 1989.

12. Ministerio de Justicia. Los derechos laborales y la disciplina ante el trabajo. La Habana: Editorial Ciencias Sociales; 1984.

13. Carrillo R, Dieste W. Tendencias actuales en el análisis económico de la morbilidad laboral. Rev Cubana Hig Epidemiol. 2000; 38(1):60-7.

14. Pérez J. Contribución al estudio de la lumbalgia inespecífica [serie en Internet]. 2006[citado 3 Ene 2007]. Disponible en:

http://bvs. sld.cu/revistas/ort/vol20 2 06/ort11206.htm

15. Cush JJ, Lipskyp E. Enfermedades de las Articulaciones. En: Harrison, editor. Principios de Medicina Interna. 13aํ ed. Vol II. España: McGraw-Hill; 1994.p.194253.

16. Farreras P. Enfermedades del Aparato Locomotor. En: Medicina Interna. $6^{\text {ta }}$ ed. España: Marín, S.A.;1962.p.1012- 80.

17. Herrero-Beumont G. Artrosis o enfermedad degenerativa articular [serie en Internet]. 2006[citado 10 Ene 2007]. Disponible en: http://bvs.sld.cu/revistas/ort/vol20_2_06/ort11206.htm

18. Álvarez R. Afecciones de la columna dorsolumbar, el tórax y la pelvis. En: Álvarez R, editor. Tratado de Cirugía Ortopédica y traumatología. T III. La Habana: Editorial Pueblo y Educación; 1986.p.218-94.

19. Kenneth D. Artrosis. En: Harrison. editor. Principios de Medicina Interna. 13a ed. España: McGraw- Hill; 1994.p.1947-53.

Recibido: 5 de febrero de 2008.

Aprobado: 23 de octubre de 2008.

Antonio Castillo Guzmán. Instituto de Cardiología y Cirugía Cardiovascular. Calle 17 entre Paseo y A. El Vedado. La Habana, Cuba.

E-mail: acastillo@icccv.sld.cu 
No. de certificados médicos

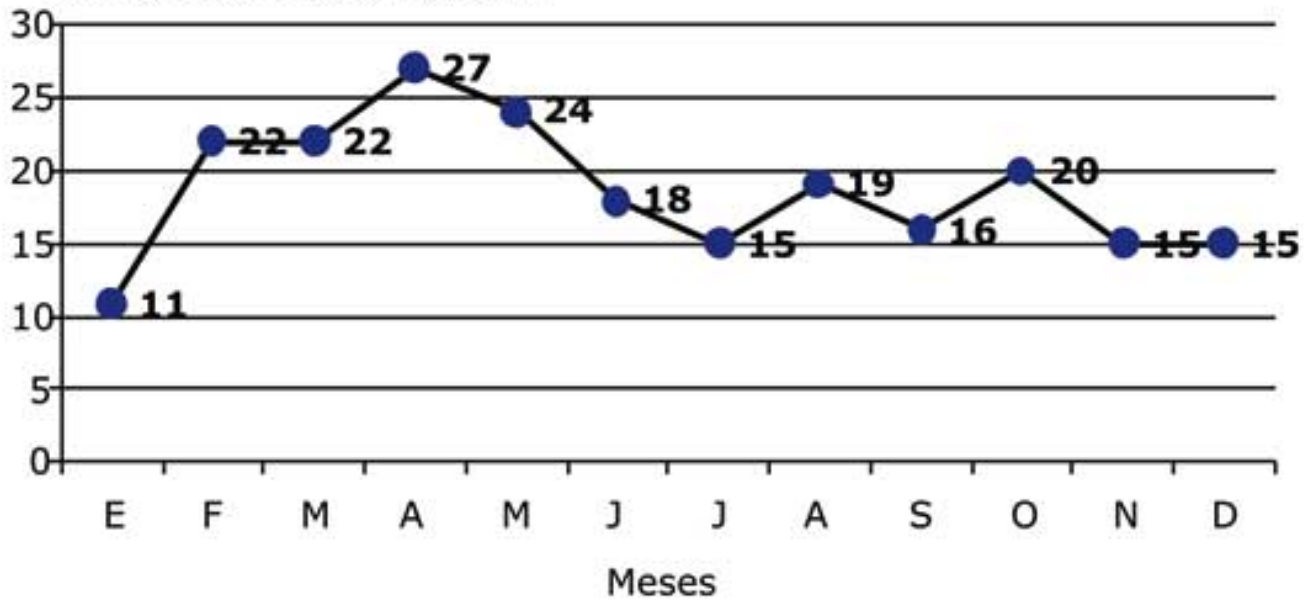

Fuente: Registro de certificados médicos. Dpto. de Recursos Humanos ICCCV.

Fig. Certificados médicos según meses del año. ICCCV 2005. 